\title{
Apigenin attenuates molecular, biochemical, and histopathological changes associated with renal impairments induced by gentamicin exposure in rats
}

Manal M. Hussein

Helwan University

Hussam A. Althagafi

Al Baha University

Fahad Alharthi

Taif University

Ashraf Albrakati

Taif University

Khalaf F. Alsharif

Taif University

Abdulrahman Theyab

Security Forces Hospital Program Makkah

Rami B. Kassab ( $\square$ rami.kassap@yahoo.com )

Helwan University Faculty of Science https://orcid.org/0000-0002-1520-1601

Ahmad H. Mufti

Umm Al-Qura University

Mohammad Algahtani

The Comprehensive Specialized Clinics

Atif Abdulwahab A. Oyouni

University of Tabuk

Roua S. Baty

Taif University

Ahmed E. Abdel Moneim

Helwan University

Maha S. Lokman

Prince Sattam bin Abdulaziz University

Research Article 
Keywords: apigenin, gentamicin, nephrotoxicity, kidney function, oxidative stress

Posted Date: February 15th, 2022

DOI: https://doi.org/10.21203/rs.3.rs-1255144/v1

License: (c) (i) This work is licensed under a Creative Commons Attribution 4.0 International License. Read Full License 


\section{Abstract}

Gentamicin (GM) is an aminoglycoside antibiotic used to treat bacterial infections. However, its application is accompanied by renal impairments. Apigenin is a flavonoid found in many edible plants with potent therapeutic values. This study was designed to elucidate the therapeutic effects of apigenin on GM-induced nephrotoxicity. Animals were injected orally with three different doses of apigenin $(5,10$ and $20 \mathrm{mg} \mathrm{kg}^{-1} \mathrm{day}^{-1}$ ). Apigenin administration abolished the alterations in the kidney index and serum levels of kidney-specific functions markers, namely blood urea nitrogen and creatinine, and KIM-1, NGAL, and cystatin C following GM exposure. Additionally, apigenin increased levels of enzymatic (glutathione reductase, glutathione peroxidase, superoxide dismutase and catalase), and non-enzymatic antioxidant proteins (reduced glutathione) and decreased levels of lipid peroxide, nitric oxide, and downregulated nitric oxide synthase- 2 in the kidney tissue following GM administration. At the molecular scope, apigenin administration was found to upregulate the mRNA expression of $N f e 2 / 2$ and $H$ mox 1 in the kidney tissue. Moreover, apigenin administration suppressed renal inflammation and apoptosis by decreasing levels of interleukin- $1 \beta$, tumor necrosis factor-alpha, nuclear factor kappa-B, Bax, and caspase- 3 , while increasing B-cell lymphoma-2 compared with those in GM- administered group. The recorded data suggests that apigenin treatment could be used to alleviate renal impairments associated with GM administration.

\section{Introduction}

Gentamicin (GM) is an aminoglycoside antibiotic commonly used for treating severe bacterial infections (Sales \&Foresto 2020). Among aminoglycosides, GM possesses the most adverse effects, including oxidative stress and renal toxicity (Morales-Alvarez 2020). Another adverse effect of GM is renal dysfunction related to GM's aggregation in the proximal tubules (Houghton et al. 1988).

Enhanced oxidative stress, inflammatory reaction, necrosis, and apoptosis in tubular cells are the most significant mechanisms in GM-induced kidney dysfunction both in vivo and in cell culture (Girton et al. 2002, Karadeniz et al. 2008a). Such complications resulted from the accumulation of GM in cells of the renal tubules and the interaction between GM and cellular organelles (Ghaznavi et al. 2018).

Renal injury may be acute or chronic and is conventionally detected by measuring the serum levels of blood urea nitrogen (BUN) and creatinine, which only increase when renal damage reaches a significant degree and usually rise at least 1 week after GM treatment initiation (Karadeniz et al. 2008a). Although serum creatinine and BUN levels are the main traditional parameters for evaluating kidney function (Josiah et al. 2020), they lack sensitivity and specificity. Kidney injury molecule 1 (KIM-1) and neutrophil gelatinase-associated lipocalin (NGAL) are recently considered more specific markers for renal injury (Ragab et al. 2014).

GM binds to phospholipids in the renal cell membranes causing the inactivation of phospholipases, leading to the development of renal disorders, such as toxicity (Rougier et al. 2004). Remarkably, flavonoid derivatives were considered nephroprotective agents because they prevent the binding of GM to 
the phospholipids in the cytoplasmic membranes (Valipour et al. 2016). In the same line, some flavonoids exert nephroprotective activities against GM nephrotoxicity (Ghaznavi et al. 2016, Karadeniz et al. 2008a).

Apigenin or 4,5,7-trihydroxyflavone is a flavonoid naturally found in fruits and vegetables (Ju et al. 2015). Apigenin has a nephroprotective effect against cisplatin nephropathy by promoting antioxidant, antiinflammatory, and anti-apoptotic pathways (Hassan et al. 2017, Malik et al. 2017). However, the therapeutic role of apigenin against GM-induced renal damage has not been elucidated. Therefore, this study evaluates the capability of apigenin in attenuating GM-induced nephrotoxicity.

\section{Material And Methods}

\section{Animals}

This study included 42 male rats weighing approximately $200-220 \mathrm{~g}$ obtained from VACSERA (Cairo, Egypt). All rats were kept in cages under an appropriate environment with a mean temperature of $25 \pm 5$ ${ }^{\circ} \mathrm{C}$ and mean humidity of $50 \pm 10 \%$ in a 12-h light/dark cycle. All animals were kept for 1 week with free water and food before the initiation of experiments that were performed according to the ethical principles of the Institutional Animal Care and Use Committee (IACUC) of Helwan University (approval no. HU2019/Z/AER919-01).

\section{Experimental protocol}

Animals were allocated into sex groups with seven rats in each, as follows: in the healthy control group, the rats received saline; in the apigenin-treated group, the rats received apigenin $\left(20 \mathrm{mg} \mathrm{kg}^{-1} \mathrm{day}^{-1}\right)$; in the GM-treated group, the rats received an intraperitoneal injection of GM (100 mg kg-1 day $\left.{ }^{-1}\right)$ (Sigma, St. Louis, MO, USA); in the apigenin-5-GM group, the rats orally received apigenin $\left(5 \mathrm{mg} \mathrm{kg}^{-1}\right.$ day $\left.^{-1}\right) 1 \mathrm{~h}$ before GM injection; in the apigenin-10-GM group, the rats orally received apigenin (10 $\left.\mathrm{mg} \mathrm{kg}^{-1} \mathrm{day}^{-1}\right) 1 \mathrm{~h}$ before GM injection; and in the apigenin-20-GM group, the rats orally received apigenin $\left(20 \mathrm{mg} \mathrm{kg}^{-1}\right.$ day $\left.^{-1}\right) 1 \mathrm{~h}$ before GM injection. The treatments were repeated daily for seven days at the same time point. Apigenin was first dissolved in dimethyl sulfoxide (DMSO) and further diluted with physiological saline $(0.9 \%$ sodium chloride). Each rat from the apigenin-treated groups received no more than $0.2 \%$ DMSO, corresponding to $10 \mu \mathrm{l}$. In the control and GM-treated groups, each rat received physiological saline with $10 \mu \mathrm{l}$ DMSO. The selected doses for GM and apigenin were based on the studies by Karadeniz et al. (2008a) and Malik et al. (2017) respectively.

\section{Sampling}

Twenty-four hours after the experiment, all animals were euthanized by intraperitoneal injection of pentobarbital $\left(300 \mathrm{mg} \mathrm{kg}^{-1}\right)$. The blood samples were collected and centrifuged for $10 \mathrm{~min}$ at $3000 \times \mathrm{g}$ to separate the serum, which was then stored at $-80^{\circ} \mathrm{C}$ for biochemical analyses. The kidneys were removed and weighed for calculation of the renal index as follows: 
Index of the kidneys $=$ the weight of the kidney $(\mathrm{g}) \times 100$

the final weight of the body $(\mathrm{g})$

A kidney was divided into two portions: the first portion was fixed in $10 \%$ neutral buffered formalin for histopathological examinations; the second portion was retained at $-80^{\circ} \mathrm{C}$ for biochemical and molecular investigations.

\section{Measurement of BUN, creatinine, sodium, and potassium}

The serum levels of creatinine, BUN, sodium, and potassium were measured using colorimetric kits following the methods performed by Biodiagnostics (Giza, Egypt).

\section{Determination of KIM-1, NGAL, and cystatin C levels}

The serum levels of KIM-1, NGAL, and cystatin C were measured using an enzyme-linked immunosorbent assay (ELISA) according to the instructions by Abcam (Cambridge, UK).

\section{Measurement of oxidative stress parameters}

The kidneys were minced and homogenized in a phosphate buffer $(10 \mathrm{mM} ; \mathrm{pH} 7.4)$. The homogenate was centrifuged at $3000 \times g$ for $10 \mathrm{~min}$. Lipid peroxide (LPO) levels were detected according to the method used by Ohkawa et al. (1979). Nitric oxide (NO) levels were investigated following the technique used by Green et al. (1982). Glutathione (GSH) level reductions were estimated using the procedure used by Ellman (1959). The activities of GSH peroxidase (GPx), GSH reductase (GR), superoxide dismutase (SOD), and catalase were analyzed using the protocol described by Paglia and Valentine (1967), De Vega et al. (2002), Nishikimi et al. (1972) and Aebi (1984) respectively.

\section{Determination of inflammatory biomarkers}

The renal levels of nuclear factor kappa B p65 subunit (NF-kB-p65), tumor necrosis factor-alpha (TNF-a), and interleukin (IL)-1 $\beta$ were assayed using ELISA kits produced by CUSABIO Life Sciences (Wuhan, China) according to the protocols of the manufacturer.

\section{Assessment of apoptotic protein markers}

The protein levels of B-cell lymphoma 2 (Bcl-2), Bax, and caspase-3 in the kidneys were measured using ELISA kits bought from CUSABIO Life Sciences (Wuhan, China).

\section{Quantitative real-time polymerase chain reaction (PCR) technique}

Total RNA was extracted from renal tissues using a TRIzol reagent kit (Qiagen, Germantown, MD, USA); then, their concentrations were measured in nanodrops. cDNA was obtained from isolated RNA using the reverse-transcription method according to the RevertAid ${ }^{\mathrm{TM}} \mathrm{H}$ Minus Reverse Transcriptase kit provided by Fermentas (Thermo Fisher Scientific Inc., Canada). mRNA levels of Nos2, Nfe212, and Hmox1 were 
quantitatively measured using the $\mathrm{ViiA}^{\mathrm{TM}} 7 \mathrm{PCR}$ system (Applied Biosystems, USA) using the SYBR Green PCR kit (Qiagen, Germany). The fold changes of all mRNAs were calculated using the $2^{-\triangle \Delta C t}$ method, where they were normalized to the Actb acting as the internal control. Primer sequences of the selected genes are presented in Table 1.

\section{Histological procedures}

Fresh isolated kidneys were cut into small pieces and placed in $10 \%$ neutral buffered formalin.

Specimens were passed through a standard alcohol dehydration-xylene sequence, embedded in paraffin, and cut into thin sections ( $5 \mu \mathrm{m}$ in thickness). Tissue sections were stained with hematoxylin and eosin to record any histological lesions (Drury \&Wallington 1981). A light microscope (Nikon Eclipse, E200-LED, Tokyo, Japan) with various magnifications was used for this purpose.

\section{Statistical analysis}

All statistical analyses were performed using Statistical Package for the Social Sciences (IBM Corp., Armonk, NY, USA), and all data were presented as mean \pm standard deviation. One-way analysis of variance was used to assess the significant difference between different groups; $p$ values less than 0.05 were used to denote statistical significance.

\section{Results}

\section{Effects of apigenin on renal index and kidney function markers}

A significant increase in the renal index was detected in the GM-treated group $(p<0.05)$ compared with the control group (Fig. 1). Conversely, apigenin-treated rats had significantly decreased renal index compared with nephrotoxic rats $(p<0.05)$; however, the renal index of rats treated with low-dose apigenin was still significantly higher than that of healthy control rats. Remarkably, the two doses $\left(10 \mathrm{mg} \mathrm{kg}^{-1}\right.$ and $20 \mathrm{mg}$ $\mathrm{kg}^{-1}$ ) of apigenin significantly altered the renal index, suggesting that apigenin restrains the increment in the renal index triggered by GM in a dose-dependent manner.

Furthermore, the levels of kidney function markers, BUN and creatinine, in different animal groups are shown in Fig.1. The GM-injected group showed a significant increase in the levels of BUN and creatinine compared with the control group $(p<0.05)$. Remarkably, a significant decrease $(p<0.05)$ in BUN and creatinine levels was detected in the three groups pretreated with apigenin (doses: 5,10 , and $20 \mathrm{mg} \mathrm{kg}^{-1}$ ) compared with the GM-treated group. Additionally, a non-significant change in the sodium and potassium levels was observed in the GM-and apigenin-treated groups compared with the control group.

Fig. 1 also displays the concentrations of KIM-1, NGAL, and cystatin C in the different groups. Serum KIM1, NGAL, and cystatin C concentrations significantly increased in the GM-treated group compared with the control group. However, treatment with the three doses of apigenin resulted in a significant decrease in the serum concentrations of KIM-1, NGAL, and cystatin compared with the GM-treated group. 


\section{Apigenin stimulates renal antioxidant mechanisms in GM-treated animals}

The injection of GM-induced oxidative stress in renal tissues is evidenced by the rising levels of NO and LPO and decreasing levels of SOD, CAT, GPx, GR, and GSH (Fig. 2). However, apigenin (5, 10, and $20 \mathrm{mg} \mathrm{kg}^{-1}$ ) possessed antioxidant properties, as shown by the elevated protein levels of SOD, CAT, GPX, $\mathrm{GR}$, and GSH, along with a significant reduction in the levels of LPO, NO, and Nos2 compared with the GM-injected group.

Furthermore, to test the effect of apigenin on the Nrf2/HO-1 pathway in the renal tissues of GM-treated rats, we examined the mRNA expression of $N f e 2 / 2$ and Hmoxin the renal tissues of the different animal groups. GM-induced a significant downregulation in the mRNA expression of $\mathrm{Nfe} / 2 \mathrm{2}$ and Hmox 1 (Fig. 3). Apigenin per se caused a significant upregulation in Nfe2/2 expression. Moreover, pretreatment with apigenin restrained the harmful effects of GM, and their expressions were upregulated after apigenin administration.

\section{Apigenin administration mitigates GM-induced renal inflammation and apoptosis}

GM-induced a pro-inflammatory response evidenced by a significant increase $(p<0.05)$ in the protein levels of NFKB-p65, IL-1 $\beta$, and TNF- $\alpha$ in the kidneys compared with those of the control group (Fig. 4). However, apigenin treatment diminished renal inflammation, as shown by a significant decrease in the protein levels of IL-1 $\beta, N F K B-p 65$, and TNF-a compared with those in the GM-treated group $(p<0.05)$.

In relation to the results in the control group, GM mediated apoptosis in the kidneys of rats, as evidenced by a significant rise $(p<0.05)$ in the protein levels of Bax and caspase- 3 along with a decline in Bcl-2 level (Fig. 4). Conversely, in the three apigenin-treated groups, apigenin could promote anti-apoptotic mechanisms by increasing Bcl-2 and decreasing Bax and caspase-3 protein levels compared with the GM-treated group.

\section{Apigenin protects renal tissue following $\mathrm{GM}$ administration}

To evaluate the nephroprotective effect of apigenin on the renal histological alternations, the renal tissues were examined using hematoxylin and eosin (H\&E) staining. As a result, the GM-treated group showed edema in the renal corpuscles with congested glomeruli, severe infiltration of inflammatory leukocytes, cytoplasmic vacuolation, and tubular epithelial injury (Fig. 5C). Pretreatment with apigenin attenuated glomerular and tubular injuries in rats with GM-induced nephrotoxicity (Figs. 5D, 5E, and 5F). The nephroprotective effects of apigenin were supported by a decrease in inflammatory cell infiltration and preservation of the glomerulus. The control and rats treated with apigenin alone showed normal morphology of the glomeruli and tubular cells (Figs.5A and 5B).

\section{Discussion}


GM-induced renal injury has been considered the most well-defined model for studying drug-associated nephrotoxicity (Karadeniz et al. 2008a). A significant increase in the renal index was observed in the current study after GM treatment, which conforms to Abdelrahman and Abdelmageed (2020) and Feyzi et al. (2020). This increase may be related to histopathological alterations, as Udupa and Prakash (2019) reported. Moreover, Karadeniz et al. (2008b) have attributed this increase to the generation of inflammatory mediators and interstitial infiltration of immune cells, followed by edema development in GM-treated animals. Interestingly, apigenin administration reduced the increased renal index in response to GM exposure.

Creatinine and BUN are considered the main traditional parameters for evaluating kidney function (Ehsani et al. 2017). In this study, a significant increase in creatinine and BUN serum levels was observed in the GM-treated group compared with the control group. These results conform to many studies that have demonstrated that over-discharge of creatinine and urea into the serum could indicate reduced glomerular filtration rate (GFR) and impaired renal function, especially proximal tubule function (Abdelrahman \&Abdelmageed 2020, Gharaei et al. 2019, Yaribeygi et al. 2019). Moreover, elevated creatinine and BUN levels were considered the most important hallmarks of GM-induced renal toxicity (Ghaznavi et al. 2018, Mestry et al. 2020). Upraised creatinine and BUN levels in this study may be explained by efficient reabsorption of GM in the proximal renal tubule, and GM's accumulation in tubular cells disturbed the renal circulation, reducing the GFR and subsequently raising serum creatinine and BUN levels (Mestry et al. 2020). On the other hand, apigenin administration caused a noticeable reduction in the serum levels of creatinine and BUN and restored their normal levels as compared to GM-treated rats. The renoprotective effect of apigenin was shown by a reduction in the serum levels of BUN and creatinine could be due to its antioxidant capabilities, as reactive oxygen species (ROS) might be responsible for reducing GFR (Pedraza-Chaverrí et al. 2000).

KIM-1 and NGAL are recently considered more specific and sensitive renal injury markers (Ragab et al. 2014, Yin et al. 2019). KIM-1 elevation may indicate renal damage, as demonstrated by Hansen et al. (2001), who reported that KIM-1 is a membranous glycoprotein unnoticeable in normal renal tissue or the urine but is overexpressed in the proximal renal tubules after renal injury. Besides, KIM-1 is used for the early sensitive and specific detection of nephrotoxicity induced by and chemicals (Al-Brakati et al. 2021, Udupa \&Prakash 2019). NGAL is a glycoprotein found at low concentrations in the proximal tubule and neutrophils (Flower et al. 2000). A recent study detected a significant increase in NGAL in the GM-treated group compared with the healthy control group. Similar to our results, a highly expressed NGAL was observed within the thick ascending loop of Henle, distal renal tubule, and collecting tubule of rats and mice with renal toxicity (Medić et al. 2019). Likewise, in vivo studies have shown that the levels of KIM-1 and NGAL altered before tubular renal injury, and these changes are coupled with the severe histopathological changes in the renal tubules proposing their efficiency in predicting GM-induced acute renal damage (Luo et al. 2016).

Cystatin $\mathrm{C}$ is a lysosomal enzyme that constrains the degradation of certain proteins within and outside a cell. This enzyme is expressed by all cells, except for nucleated ones, and is formed and carried into the 
plasma at a stable level in healthy conditions (Grubb 1992). In this study, a substantial upregulation of cystatin $\mathrm{C}$ was observed in GM-treated rats compared with the healthy control group. These observations conform to the study of Udupa and Prakash (2019), who have attributed this increase to the cellular degradation of the proximal tubules and diminished reabsorptive ability. Furthermore, cystatin $\mathrm{C}$ was used for detecting acute damage in the renal tubules and glomeruli in experimental animals, and it was considered a highly sensitive biomarker (Dieterle et al. 2010).

It was indicated that treatment of experimental animals with GM elicited renal dysfunction depending on its accumulation in cells of the renal tubules (Houghton et al. 1988). Several studies have proposed that ROS is known to be the master mediator of GM-induced acute renal injury (Karadeniz et al. 2008a). GM induces nephrotoxicity by overproducing ROS, triggering lipid peroxidation of cytoplasmic membranes and protein degradation. These severe changes consequently resulted in improper enzymatic activation, mitochondrial function, and cellular damage (Ghaznavi et al. 2018, Ghaznavi et al. 2016, Mestry et al. 2020).

NO acts as a signal substance and guardian of cell functions as it plays an important role in maintaining normal physiology of the kidney by controlling both blood flow in the renal cortex and function of the renal tubules (Fujihara et al. 2006, Kassab et al. 2021). In addition, NO regulates signal transduction pathways, cells energetics, host immunoreactions, and the pathology of kidney failure (Yousefipour et al. 2010). In this study, the level of NO increased in the kidneys of GM-treated rats, and these results conform to other studies (El-Kashef et al. 2016). In this study, the overproduction of NO may be due to a hyperactive inducible NO synthase (iNOS) that induces NO production (Al-Brakati et al. 2020). NO overproduction can induce cell damage by reacting with superoxide anion $\mathrm{O}_{2}{ }^{-}$generating cytotoxic peroxynitrite. In other words, NO and peroxynitrite act as key mediators of oxidative stress and pathophysiology in GM-induced nephrotoxicity (Passauer et al. 2005). They cause several adverse effects, such as protein degradation, enzymatic inactivation, peroxidation of lipids, disruption of the respiratory chain in cells, and impairment of DNA repair systems (Al-Brakati et al. 2020). Our data showed that NO levels decreased after apigenin treatment, and this observation was confirmed by Al-Brakati et al. (2020), who have reported that flavonoid-derived compounds prevent the expression of iNOS and its isoforms, which are responsible for the production of $\mathrm{NO}$ and pro-inflammatory cytokines.

The formation of renal LPO in this study conforms to previous studies (Ghaznavi et al. 2016, Kang et al. 2013). LPO increases cytoplasmic membranes' permeability, resulting in ion-exchange imbalance (Kang et al. 2013). In normal conditions, antioxidant enzymes, such as SOD, CAT, GPx, and GR, can reduce renal damage by scavenging free radicals or ROS and preventing GM-induced apoptosis (Martínez-Salgado et al. 2004, Randjelovic et al. 2012). In this study, the decreased levels of SOD, CAT, GR, and GPx observed in GM-treated rats may be attributed to GM-induced ROS generation that inhibits the defense mechanisms elicited by these enzymes (Martínez-Salgado et al. 2004, Randjelovic et al. 2012). GM-induced oxidative injury in this study conforms to those indicated by several studies (Daenen et al. 2019, Marinho et al. 2020), which have clarified that disruption between massive free radicals and deficient degradation of these radicals by antioxidant defenders might be the reason for GM-induced renal injury. 
Remarkably, the increased LPO levels and decreased antioxidants concentrations were noticeably retained by apigenin treatment. These results conform to several studies (Hassan et al. 2017, Wang et al. 2014), which have indicated that apigenin exerts a nephroprotective effect by presenting antioxidant properties, allowing it to scavenge several free radicals, preventing LPO formation and restoring antioxidant enzyme levels to normal.

Heme oxygenase $1(\mathrm{HO}-1)$ is a rate-limiting protein in the catabolic pathway of heme, which converts into bilirubin by liberating iron and carbon monoxide. HO-1 with other antioxidant enzymes limits redox imbalance; thus, it is activated to respond to oxidative stress (Abraham \&Kappas 2008). Hmox 1 is a target gene of nuclear factor erythroid-2-related factor 2 ( $N f e 2 / 2$ or Nrf2) that regulates its expression (AlBrakati et al. 2020). This regulation can be explained as follow: Nrf2 is considered a cell defender factor that is enhanced in response to ROS through translocation into the nucleus and binding to an antioxidant response element (ARE) that in turn triggers the transcription of Hmox 1 and other antioxidant genes (Mills et al. 2018).

In the same line, Nrf2 is involved in various cellular protective mechanisms, such as mediating antioxidative mechanisms in various renal diseases (Nezu \&Suzuki 2020). The upregulation of Hmox 1 and $N f e 212$ mRNAs after apigenin treatment observed in this study was also observed in rat primary hepatocytes, as shown by Huang et al. (2013), who have reported that the overexpression of these genes could inhibit oxidative stress induced by tert-butyl hydroperoxide. Additionally, Yang et al.(2018a) have indicated that treatment of high-fructose diet-fed mice with apigenin enabled the translocation of $\mathrm{Nfe} 212$ into the nucleus and the subsequent rise of Hmox 1 expression led to the alleviation of oxidative stress. Similar results were observed in ischemic male rats treated with apigenin in a study by Zhang et al. (2019), who concluded that apigenin acts as a powerful antioxidant.

Apoptosis plays a key role in the normal physiology of the renal functions and in drug-induced nephrotoxicity, where dysregulation of apoptotic mechanisms results in various renal disorders (Ansari et al. 2016). Apoptosis is initiated by a cascade of caspase-1, caspase-8, and caspase-9 that trigger caspase-3, whose activation is the key mediator of GM-induced renal cell death (Chen et al. 2011). Bcl-2 is the main apoptotic inhibitor that is considerably underexpressed in injured renal tissues (Meier et al. 2000)

Currently, the GM-treated group displayed an elevation in caspase-3 and Bax levels and a decrease in Bcl2 levels; our data conform to other studies.(Abdelrahman \&Abdelmageed 2020, Yang et al. 2018b). Apoptosis detected in GM-treated rats of this study may be due to several mechanisms, including the accumulated GM within the endoplasmic reticulum preventing RNA translation into proteins and constrained the posttranslational processing of proteins (Horibe et al. 2004). These mysterious events put the endoplasmic reticulum under stress and stimulated cell death (Peyrou et al. 2007). Additionally, GM may interfere with the transport function of some transmembrane proteins, such as the sodiumpotassium pump (Sassen et al. 2006), resulting in improper tubular reabsorption, cell swelling, and substantial necrosis or programmed cell death (Morales-Alvarez 2020). 
In addition, apoptosis observed after GM administration in this study may be due to the overproduction of ROS, as was indicated in other studies (Bustos et al. 2016, Ehsani et al. 2017), that recorded a significant relationship between oxidative stress and the induction and progression of renal cell death in many experimental animals. This relationship can be explained as follow: GM-induced ROS can activate intrinsic pathways of mitochondria by disturbing cellular respiration machinery and reducing ATP formation and triggers the liberation of cytochrome $C$ and the overexpression of other pro-apoptotic proteins, such as Bax, causing cleavage of key proteins, nuclear envelope, and DNA, resulting in apoptosis (Bustos et al. 2016). Importantly, in this study, apigenin could attenuate GM-induced apoptosis by decreasing renal caspase-3 and Bax levels and increasing Bcl-2 levels. Similarly, in vivo studies, apigenin treatment could reverse renal alterations caused by apoptosis in animals with cisplatin-induced nephrotoxicity and renal ischemia-reperfusion injury (Hassan et al. 2017, Liu et al. 2017).

Cytokines are inflammatory mediators that regulate normal cellular physiology and are associated with tissue damage and repair (Ramesh \&Reeves 2004). GM-induced nephrotoxicity has also been associated with the activation and excessive release of inflammatory cytokines, predominantly IL-1 $\beta$ and TNF- $a$ (Mahmoud 2017, Salama et al. 2018). In this study, the significant increase in the renal levels of NFKBp65 and TNF-a observed in the GM-treated group was also indicated in other studies revealing that NFKBp65 overstimulation is accompanied by an aggregation of TNF-a (El-Kashef et al. 2016, Sahu et al. 2014). Additionally, NF-KB is considered a principal initiator of inflammatory reactions, especially renal inflammation, in GM-induced renal toxicity (Bae et al. 2014). Furthermore, TNF- $a$ is the main regulator of the renal immune response triggered by many drugs by amplifying renal pathophysiological mechanisms elicited by drugs (Fredriksson et al. 2011).

The increased level of IL-1 $\beta$ recorded in this study was also observed by Bae et al. (2008), who have elucidated that GM activates NFKB-p65, which subsequently resulted in the overproduction of proinflammatory cytokines. The reduction of the protein levels of NFKB-p65, IL-1 $\beta$, and TNF- $\alpha$ after apigenin treatment recorded in this study could be attributed to the anti-inflammatory ability of apigenin, which was also observed in cisplatin-induced nephrotoxicity models in a study by Funakoshi-Tago et al. (2011), who have observed that apigenin could overwhelm the inflammatory response immunomodulation in the kidneys by diminishing NFKB-p65, IL-1 $\beta$, and TNF-a levels.

The anti-inflammatory effect exerted in the apigenin-treated groups may be due to apigenin's capability of interfering with the NF-KB signaling pathway through several molecular mechanisms, such as direct inhibition of signal transducer and activator of transcription 3 , blockage of the phosphorylation and degradation of inhibitor of NF-KB, and inactivation of inhibitor of NF-KB kinase (Shukla et al. 2015). These adverse events, in turn, resulted in the inactivation of NF-KB (Qin et al. 2016), improper translocations to the nucleus, and loss of DNA-binding activity (Wang et al. 2014).

\section{Conclusion}


Collectively, apigenin administration normalized kidney index and kidney function markers following GM exposure. Additionally, apigenin prevented the renal oxidative damage by decreasing pro-oxidant and enhancing Nrf2 and its downstream antioxidant proteins. Moreover, apigenin suppressed the inflammatory and apoptotic cascades induced by GM. These results suggest that apigenin could use to alleviate the renal impairments associated with GM application.

\section{Declarations}

Funding: Not applicable.

Acknowledgements: The authors thank the Taif University Researchers Supporting Program (Project number: TURSP-2020/151), Taif University, Saudi Arabia, for supporting this study.

Data availability: All relevant data are within the paper.

\section{Ethics approval}

All procedures were performed according to the ethical principles of the Institutional Animal Care and Use Committee (IACUC) of Helwan University (approval no. HU2019/Z/AER919-01).

Consent to participate Not applicable.

Consent to publish Consented.

Conflict of interest The author declares no competing interest.

\section{References}

1. Abdelrahman RS, Abdelmageed ME (2020) Renoprotective effect of celecoxib against gentamicininduced nephrotoxicity through suppressing NFKB and caspase-3 signaling pathways in rats. Chemico-Biol Interact 315:108863

2. Abraham NG, Kappas A (2008) Pharmacological and clinical aspects of heme oxygenase. Pharmacol Rev 60:79-127

3. Aebi H (1984) [13] Catalase in vitro, Methods in enzymology. Elsevier, pp 121-126

4. Al-Brakati A, Albarakati AJA, Lokman MS, Theyab A, Algahtani M, Menshawi S, AlAmri OD, Al Omairi NE, Essawy EA, Kassab RB, Abdel Moneim AE (2020) : Possible Role of Kaempferol in Reversing Oxidative Damage, Inflammation, and Apoptosis-Mediated Cortical Injury Following Cadmium Exposure. Neurotoxicity research

5. Al-Brakati A, Alsharif KF, Alzahrani KJ, Kabrah S, Al-Amer O, Oyouni AA, Habotta OA, Lokman MS, Bauomy AA, Kassab RB (2021) Using Green Biosynthesized Lycopene-Coated Selenium Nanoparticles to Rescue Renal Damage in Glycerol-Induced Acute Kidney Injury in Rats. Int J Nanomed 16:4335 
6. Ansari MA, Raish M, Ahmad A, Ahmad SF, Mudassar S, Mohsin K, Shakeel F, Korashy HM, Bakheet SA (2016) Sinapic acid mitigates gentamicin-induced nephrotoxicity and associated oxidative/nitrosative stress, apoptosis, and inflammation in rats. Life Sci 165:1-8

7. Bae EH, Kim IJ, Joo SY, Kim EY, Choi JS, Kim CS, Ma SK, Lee J, Kim SW (2014) Renoprotective effects of the direct renin inhibitor aliskiren on gentamicin-induced nephrotoxicity in rats. Journal of the Renin-Angiotensin-Aldosterone System 15:348-361

8. Bae WK, Lee J, Park JW, Bae EH, Ma SK, Kim SH, Kim SW (2008) Decreased expression of Na+/K+ATPase, NHE3, NBC1, AQP1 and OAT in gentamicin-induced nephropathy. The Korean Journal of Physiology \& Pharmacology 12:331-336

9. Bustos PS, Deza-Ponzio R, Páez PL, Albesa I, Cabrera JL, Virgolini MB, Ortega MG (2016) Protective effect of quercetin in gentamicin-induced oxidative stress in vitro and in vivo in blood cells. Effect on gentamicin antimicrobial activity. Environ Toxicol Pharmacol 48:253-264

10. Chen Y-C, Chen C-H, Hsu Y-H, Chen T-H, Sue Y-M, Cheng C-Y, Chen T-W (2011) Leptin reduces gentamicin-induced apoptosis in rat renal tubular cells via the PI3K-Akt signaling pathway. Eur $\mathrm{J}$ Pharmacol 658:213-218

11. Daenen K, Andries A, Mekahli D, Van Schepdael A, Jouret F, Bammens B (2019) Oxidative stress in chronic kidney disease. Pediatric nephrology 34:975-991

12. De Vega L, Fernández RP, Martin Mateo M, Bustamante JB, Herrero AM, Munguira EB (2002) Glutathione determination and a study of the activity of glutathione-peroxidase, glutathionetransferase, and glutathione-reductase in renal transplants. Ren Fail 24:421-432

13. Dieterle F, Perentes E, Cordier A, Roth DR, Verdes P, Grenet O, Pantano S, Moulin P, Wahl D, Mahl A (2010) Urinary clusterin, cystatin C, $\beta 2$-microglobulin and total protein as markers to detect druginduced kidney injury. Nat Biotechnol 28:463-469

14. Drury RAD, Wallington EA (1981) Carleton's Histological Technique. Oxford University Press, New York

15. Ehsani V, Amirteimoury M, Taghipour Z, Shamsizadeh A, Bazmandegan G, Rahnama A, Khajehasani F, Fatemi I (2017) Protective effect of hydroalcoholic extract of Pistacia vera against gentamicininduced nephrotoxicity in rats. Ren Fail 39:519-525

16. El-Kashef DH, El-Kenawi AE, Rahim MA, Suddek GM, Salem HA (2016) Agmatine improves renal function in gentamicin-induced nephrotoxicity in rats. Can J Physiol Pharmacol 94:278-286

17. Ellman GL (1959) Tissue sulfhydryl groups. Arch Biochem Biophys 82:70-77

18. Feyzi R, Yari S, Karamian R, Hasanein P (2020) : Preventive effect of Stachys lavandulifolia against gentamicin-induced oxidative stress and nephrotoxicity in rats.Comparative Clinical Pathology,1-9

19. Flower DR, North AC, Sansom CE (2000) The lipocalin protein family: structural and sequence overview. Biochimica et Biophysica Acta (BBA)-Protein Structure and Molecular Enzymology. 1482:9-24

20. Fredriksson L, Herpers B, Benedetti G, Matadin Q, Puigvert JC, de Bont H, Dragovic S, Vermeulen NP, Commandeur JN, Danen E (2011) Diclofenac inhibits tumor necrosis factor-a-induced nuclear factor- 
KB activation causing synergistic hepatocyte apoptosis. Hepatology 53:2027-2041

21. Fujihara CK, Sena CR, Malheiros DM, Mattar AL, Zatz R (2006) Short-term nitric oxide inhibition induces progressive nephropathy after regression of initial renal injury. American Journal of Physiology-Renal Physiology 290:F632-F640

22. Funakoshi-Tago M, Nakamura K, Tago K, Mashino T, Kasahara T (2011) Anti-inflammatory activity of structurally related flavonoids, Apigenin, Luteolin and Fisetin. Int Immunopharmacol 11:1150-1159

23. Gharaei FK, Safari T, Niazi AA, Bujani MZ (2019) : Losartan and magnesium sulfate administration reduce gentamicin-induced nephrotoxicity in rat model.Journal of Nephropathology 8

24. Ghaznavi H, Mehrzadi S, Dormanesh B, Tabatabaei SMTH, Vahedi H, Hosseinzadeh A, PazokiToroudi H, Rashidian A (2016) Comparison of the protective effects of melatonin and silymarin against gentamicin-induced nephrotoxicity in rats. Journal of evidence-based complementary \& alternative medicine 21:NP49-NP55

25. Ghaznavi H, Fatemi I, Kalantari H, Hosseini Tabatabaei SMT, Mehrabani M, Gholamine B, Kalantar M, Mehrzadi S, Goudarzi M (2018) Ameliorative effects of gallic acid on gentamicin-induced nephrotoxicity in rats. J Asian Nat Prod Res 20:1182-1193

26. Girton RA, Sundin DP, Rosenberg ME (2002) Clusterin protects renal tubular epithelial cells from gentamicin-mediated cytotoxicity. American Journal of Physiology-Renal Physiology 282:F703F709

27. Green LC, Wagner DA, Glogowski J, Skipper PL, Wishnok JS, Tannenbaum SR (1982) Analysis of nitrate, nitrite, and [15N] nitrate in biological fluids. Anal Biochem 126:131-138

28. Grubb A (1992) Diagnostic value of analysis of cystatin C and protein $\mathrm{HC}$ in biological fluids. Clin Nephrol 38:S20-S27

29. Hassan SM, Khalaf MM, Sadek SA, Abo-Youssef AM (2017) Protective effects of apigenin and myricetin against cisplatin-induced nephrotoxicity in mice. Pharm Biol 55:766-774

30. Horibe T, Matsui H, Tanaka M, Nagai H, Yamaguchi Y, Kato K, Kikuchi M (2004) Gentamicin binds to the lectin site of calreticulin and inhibits its chaperone activity. Biochem Biophys Res Commun 323:281-287

31. Houghton DC, English J, Benett WM (1988) Chronic tubufointerstitial nephritis and renal insufficiency associated with long-term "subtherapeutic" gentamicin. J Lab Clin Med 112:694-703

32. Huang C-S, Lii C-K, Lin A-H, Yeh Y-W, Yao H-T, Li C-C, Wang T-S, Chen H-W (2013) Protection by chrysin, apigenin, and luteolin against oxidative stress is mediated by the Nrf2-dependent upregulation of heme oxygenase 1 and glutamate cysteine ligase in rat primary hepatocytes. Arch Toxicol 87:167-178

33. Josiah SS, Crown OO, Akinmoladun AC, Olaleye MT (2020) Renoprotective property of the flavonoidrich extract of Kigelia africana fruits on gentamicin-induced nephrotoxicity in rats. Comp Clin Pathol 29:815-828

34. Ju SM, Kang JG, Bae JS, Pae HO, Lyu YS, Jeon BH (2015) : The flavonoid apigenin ameliorates cisplatin-induced nephrotoxicity through reduction of p53 activation and promotion of PI3K/Akt 
pathway in human renal proximal tubular epithelial cells. Evidence-based complementary and alternative medicine 2015

35. Kang C, Lee H, Hah D-Y, Heo JH, Kim CH, Kim E, Kim JS (2013) Protective effects of Houttuynia cordata Thunb. on gentamicin-induced oxidative stress and nephrotoxicity in rats. Toxicological research 29:61-67

36. Karadeniz A, Yildirim A, Simsek N, Kalkan Y, Celebi F (2008a) Spirulina platensis protects against gentamicin-induced nephrotoxicity in rats. Phytother Res 22:1506-1510

37. Karadeniz A, Yildirim A, Simsek N, Kalkan Y, Celebi F (2008b) Spirulina platensis protects against gentamicin-induced nephrotoxicity in rats. Phytotherapy Research: An International Journal Devoted to Pharmacological and Toxicological Evaluation of Natural Product Derivatives 22:1506-1510

38. Kassab RB, Theyab A, Al-Ghamdy AO, Algahtani M, Mufti AH, Alsharif KF, Abdella EM, Habotta OA, Omran MM, Lokman MS (2021) Protocatechuic acid abrogates oxidative insults, inflammation, and apoptosis in liver and kidney associated with monosodium glutamate intoxication in rats.

Environmental Science and Pollution Research, pp 1-14

39. Liu Y, Liu X, Wang L, Du Y, Chen Z, Chen H, Guo J, Weng X, Wang X, Wang M (2017) Effects of apigenin on the expression levels of $B$-cell lymphoma-2, Fas and Fas ligand in renal ischemiareperfusion injury in rats. Experimental and therapeutic medicine 14:5345-5354

40. Luo Q-H, Chen M-L, Chen Z-L, Huang C, Cheng A-C, Fang J, Tang L, Geng Y (2016) Evaluation of KIM1 and NGAL as early indicators for assessment of gentamycin-induced nephrotoxicity in vivo and in vitro. Kidney and Blood Pressure Research 41:911-918

41. Mahmoud YI (2017) Kiwi fruit (Actinidia deliciosa) ameliorates gentamicin-induced nephrotoxicity in albino mice via the activation of Nrf2 and the inhibition of NF-KB (Kiwi \& gentamicin-induced nephrotoxicity). Biomed Pharmacother 94:206-218

42. Malik S, Suchal K, Khan SI, Bhatia J, Kishore K, Dinda AK, Arya DS (2017) Apigenin ameliorates streptozotocin-induced diabetic nephropathy in rats via MAPK-NF-kappaB-TNF-alpha and TGF-beta1MAPK-fibronectin pathways. Am J Physiol Renal Physiol 313:F414-F422

43. Marinho AD, Silveira JA, Chaves-Filho AJ, Macedo DS, Carmo LD, Alencar NM, Costa PH, Lopes PL, Nogueira-Junior FA, Alves NT (2020) Protective Effects Of A Lipid Transfer Protein Isolated from Morinda citrifolia Seeds in Gentamicin-Induced Nephrotoxicity in Rats. Revista Brasileira de Farmacognosia 30:568-576

44. Martínez-Salgado C, Eleno N, Morales Al, Pérez-Barriocanal F, Arévalo M, López-Novoa JM (2004) Gentamicin treatment induces simultaneous mesangial proliferation and apoptosis in rats. Kidney Int 65:2161-2171

45. Medić B, Stojanović M, Rovčanin B, Kekić D, Škodrić SR, Jovanović GB, Vujović KS, Divac N, Stojanović R, Radenković M (2019) Pioglitazone attenuates kidney injury in an experimental model of gentamicin-induced nephrotoxicity in rats. Sci Rep 9:1-10

46. Meier P, Finch A, Evan G (2000) Apoptosis in development. Nature 407:796-801 
47. Mestry SN, Gawali NB, Pai SA, Gursahani MS, Dhodi JB, Munshi R, Juvekar AR (2020) Punica granatum improves renal function in gentamicin-induced nephropathy in rats via attenuation of oxidative stress. Journal of Ayurveda and integrative medicine 11:16-23

48. Mills EL, Ryan DG, Prag HA, Dikovskaya D, Menon D, Zaslona Z, Jedrychowski MP, Costa AS, Higgins $M$, Hams $E$ (2018) Itaconate is an anti-inflammatory metabolite that activates Nrf2 via alkylation of KEAP1. Nature 556:113-117

49. Morales-Alvarez MC (2020) Nephrotoxicity of Antimicrobials and Antibiotics. Adv Chronic Kidney Dis 27:31-37

50. Nezu M, Suzuki N (2020) Roles of Nrf2 in Protecting the Kidney from Oxidative Damage. Int J Mol Sci 21:2951

51. Nishikimi M, Rao NA, Yagi K (1972) The occurrence of superoxide anion in the reaction of reduced phenazine methosulfate and molecular oxygen. Biochem Biophys Res Commun 46:849-854

52. Ohkawa H, Ohishi N, Yagi K (1979) Assay for lipid peroxides in animal tissues by thiobarbituric acid reaction. Anal Biochem 95:351-358

53. Paglia DE, Valentine WN (1967) Studies on the quantitative and qualitative characterization of erythrocyte glutathione peroxidase. J Lab Clin Med 70:158-169

54. Passauer J, Pistrosch F, Büssemaker E (2005) Nitric oxide in chronic renal failure. Kidney Int 67:1665-1667

55. Pedraza-Chaverrí J, Maldonado PD, Medina-Campos ON, Olivares-Corichi IM, Hernández-Pando R, Ibarra-Rubio MaE (2000) : Garlic ameliorates gentamicin nephrotoxicity: relation to antioxidant enzymes. Free Radical Biology and Medicine 29, 602-611

56. Peyrou M, Hanna PE, Cribb AE (2007) Cisplatin, gentamicin, and p-aminophenol induce markers of endoplasmic reticulum stress in the rat kidneys. Toxicol Sci 99:346-353

57. Qin Y, Zhao D, Zhou H-g, Wang X-h, Zhong W-I, Chen S, Gu W-g, Wang W, Zhang C-h, Liu Y-r (2016) Apigenin inhibits NF-KB and snail signaling, EMT and metastasis in human hepatocellular carcinoma. Oncotarget 7:41421

58. Ragab D, Abdallah DM, El-Abhar HS (2014) : Cilostazol renoprotective effect: Modulation of PPAR- $\gamma$, NGAL, KIM-1 and IL-18 underlies its novel effect in a model of ischemia-reperfusion.Plos one9, e95313

59. Ramesh G, Reeves WB (2004) Salicylate reduces cisplatin nephrotoxicity by inhibition of tumor necrosis factor-a. Kidney Int 65:490-498

60. Randjelovic P, Veljkovic S, Stojiljkovic N, Jankovic-Velickovic L, Sokolovic D, Stoiljkovic M, llic I (2012) : Salicylic acid attenuates gentamicin-induced nephrotoxicity in rats. The Scientific World Journal 2012

61. Rougier F, Claude D, Maurin M, Maire P (2004) Aminoglycoside nephrotoxicity. Current Drug TargetsInfectious Disorders 4:153-162 
62. Sahu BD, Tatireddy S, Koneru M, Borkar RM, Kumar JM, Kuncha M, Srinivas R, Sistla R (2014) Naringin ameliorates gentamicin-induced nephrotoxicity and associated mitochondrial dysfunction, apoptosis and inflammation in rats: possible mechanism of nephroprotection. Toxicol Appl Pharmcol 277:8-20

63. Salama SA, Arab HH, Maghrabi IA (2018) Troxerutin down-regulates KIM-1, modulates p38 MAPK signaling, and enhances renal regenerative capacity in a rat model of gentamycin-induced acute kidney injury. Food Funct 9:6632-6642

64. Sales GTM, Foresto RD (2020) Drug-induced nephrotoxicity. Revista da Associação Médica Brasileira 66:s82-s90

65. Sassen MC, Kim S, Kwon T-H, Knepper MA, Miller RT, Frøkiær J, Nielsen S (2006) Dysregulation of renal sodium transporters in gentamicin-treated rats. Kidney Int 70:1026-1037

66. Shukla S, Shankar E, Fu P, MacLennan GT, Gupta S (2015) : Suppression of NF-kB and NF-кBregulated gene expression by apigenin through IKBa and IKK pathway in TRAMP mice.Plos one10, e0138710

67. Udupa V, Prakash V (2019) Gentamicin induced acute renal damage and its evaluation using urinary biomarkers in rats. Toxicology Reports 6:91-99

68. Valipour P, Heidarian E, Khoshdel A, GHOLAMI AM (2016) : Protective effects of hydroalcoholic extract of Ferulago angulata against gentamicin-induced nephrotoxicity in rats

69. Wang J, Liu Y-T, Xiao L, Zhu L, Wang Q, Yan T (2014) Anti-inflammatory effects of apigenin in lipopolysaccharide-induced inflammatory in acute lung injury by suppressing COX-2 and NF-kB pathway. Inflammation 37:2085-2090

70. Yang M, Jiang Z-h, Li C-g, Zhu Y-j, Li Z, Tang Y-z, Ni C-I (2018a) Apigenin prevents metabolic syndrome in high-fructose diet-fed mice by Keap1-Nrf2 pathway. Biomed Pharmacother 105:12831290

71. Yang Q, Zhou Y, Yin H, Li H, Zhou M, Sun G, Cao Z, Man R, Wang H, Li J (2018b) PINK1 protects against gentamicin-induced sensory hair cell damage: possible relation to induction of autophagy and inhibition of p53 signal pathway. Front Mol Neurosci 11:403

72. Yaribeygi H, Simental-Mendía LE, Butler AE, Sahebkar A (2019) Protective effects of plant-derived natural products on renal complications. J Cell Physiol 234:12161-12172

73. Yin M, Jiang N, Guo L, Ni Z, Al-Brakati AY, Othman MS, Moneim AEA, Kassab RB (2019) Oleuropein suppresses oxidative, inflammatory, and apoptotic responses following glycerol-induced acute kidney injury in rats. Life Sci 232:116634

74. Yousefipour Z, Oyekan A, Newaz M (2010) Interaction of oxidative stress, nitric oxide and peroxisome proliferator activated receptor $y$ in acute renal failure. Pharmacol Ther 125:436-445

\section{Tables}

Table 1. Primer sequences of genes analyzed in Real Time-PCR 


\begin{tabular}{|llll|}
\hline Name & Accession number & Sense (5'-3') & Antisense (5'-3') \\
\hline Actb & NM_031144.3 & GCAGGAGTACGATGAGTCCG & ACGCAGCTCAGTAACAGTCC \\
\hline Nfe2/2 & NM_031789.2 & CAGCATGATGGACTTGGAATTG & GCAAGCGACTCATGGTCATC \\
\hline Hmox1 & NM_012580.2 & TTAAGCTGGTGATGGCCTCC & GTGGGGCATAGACTGGGTTC \\
\hline Nos2 & NM_012611.3 & GGTGAGGGGACTGGACTTTTAG & TTGTTGGGCTGGGAATAGCA \\
\hline
\end{tabular}

The abbreviations of the genes: Nfe2/2: Nuclear factor-erythroid 2-related factor 2; Hmox1: Heme oxygenase 1; Nos2: nitric oxide synthase 2.

\section{Figures}
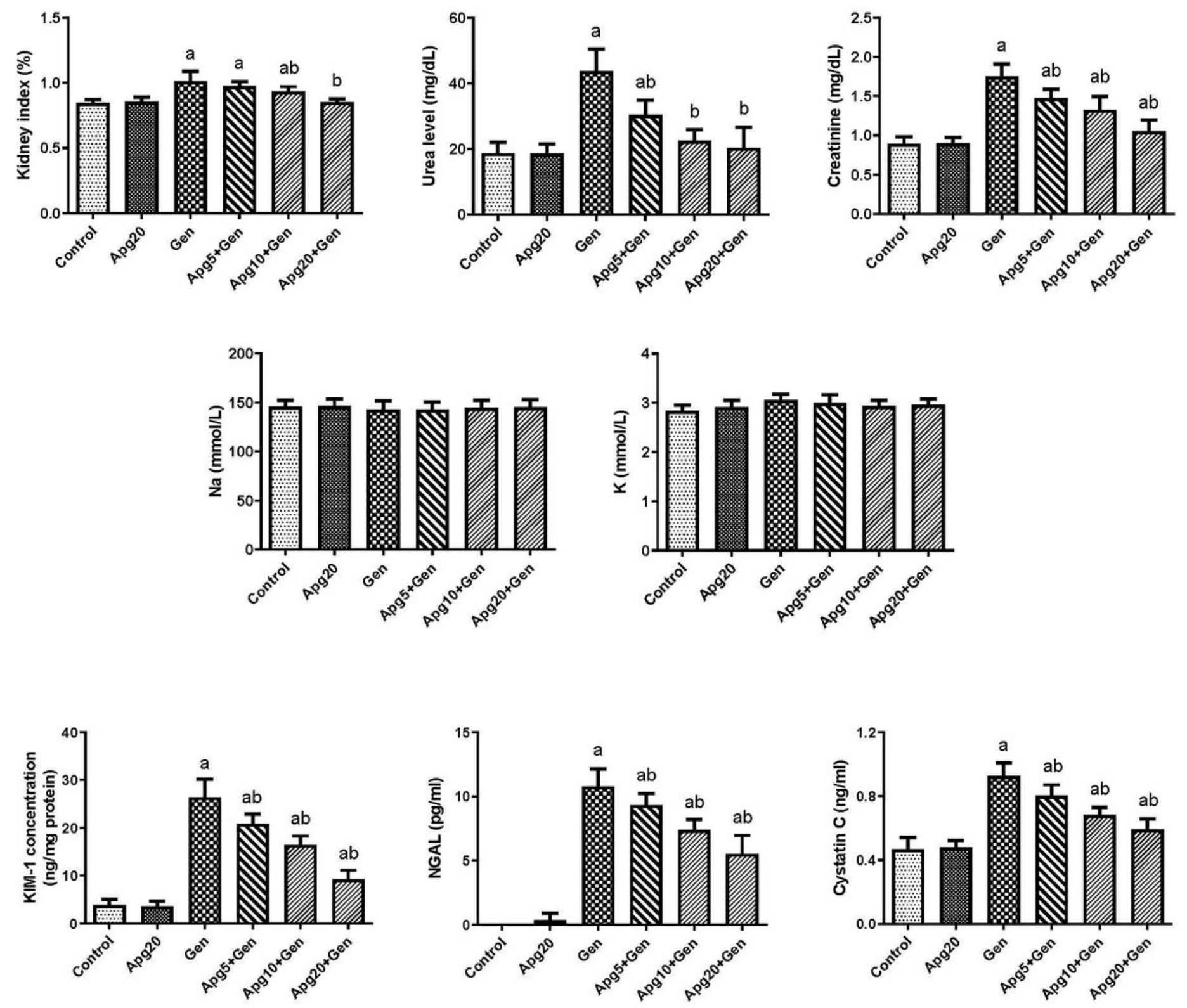
Figure 1

The renal index and blood urea nitrogen, creatinine, sodium, potassium, kidney injury molecule- 1 , neutrophil gelatinase-associated lipocalin, and cystatin $C$ levels following apigenin treatment $(5,10$, and $20 \mathrm{mg} \mathrm{kg}^{-1}$ ) in rats with gentamicin (GM)-induced nephrotoxicity. Data are expressed as mean \pm standard deviation $(\mathrm{n}=7) .{ }^{\mathrm{a}}$ represents the statistical significance relative to that of the control group at $p<0.05 .{ }^{\mathrm{b}}$ represents the statistical significance relative to that of the GM-treated group at $p<0.05$.
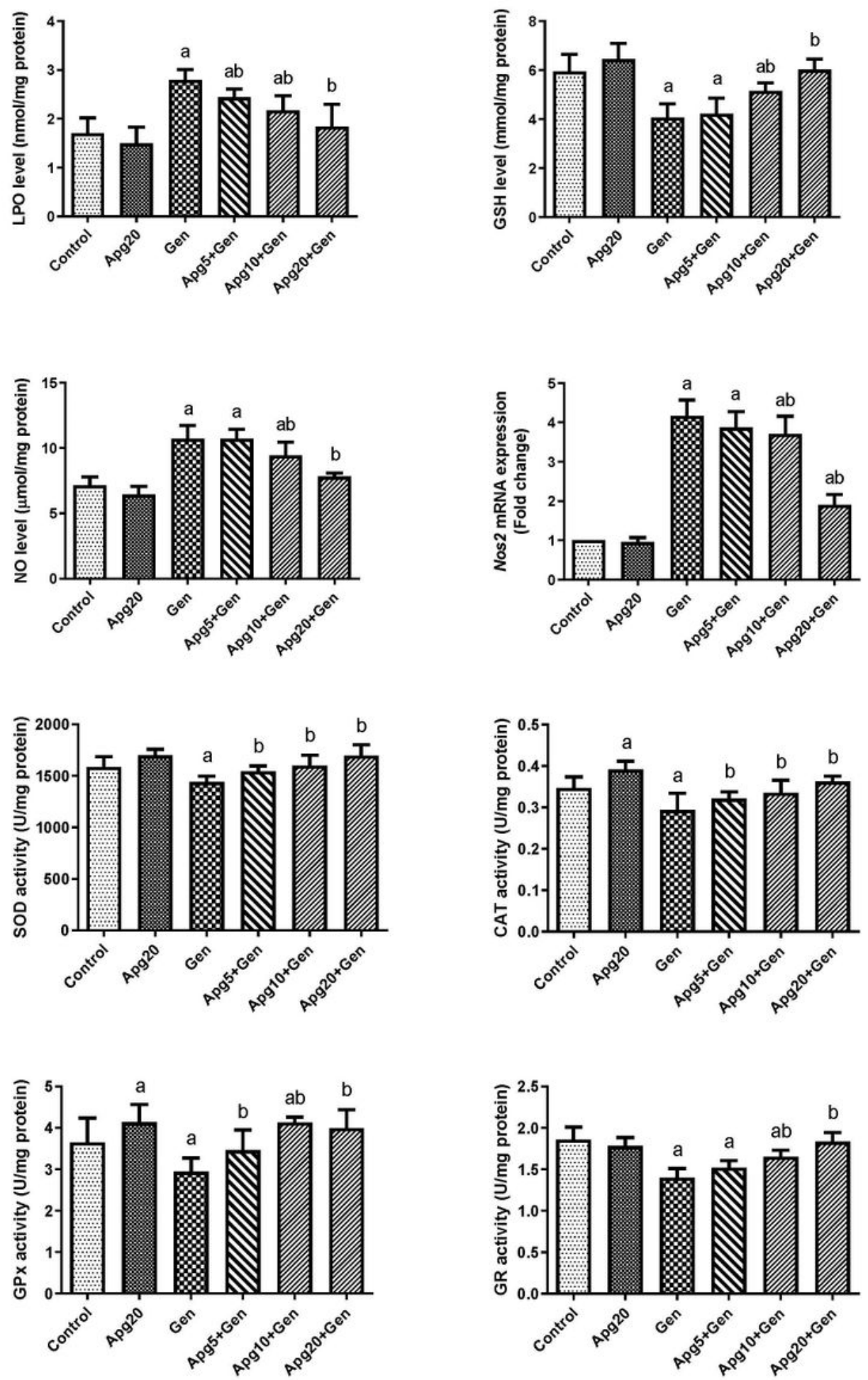
Figure 2

The renal levels of lipid peroxidation, nitric oxide (NO), NO synthase 2 (Nos2) expression, glutathione, superoxide dismutase, catalase, glutathione peroxidase, and glutathione reductase following apigenin treatment $\left(5,10\right.$, and $\left.20 \mathrm{mg} \mathrm{kg}^{-1}\right)$ in rats with gentamicin (GM)-induced nephrotoxicity. Data are expressed as mean \pm standard deviation (SD) $(n=7)$. mRNA expression results are expressed as mean \pm $\mathrm{SD}$ of three assays in duplicate references to $A c t b$ and represented as fold changes (log2 scale) compared with the mRNA levels of the control group. ${ }^{\text {a }}$ represents the statistical significance relative to that of the control group at $p<0.05 .{ }^{\mathrm{b}}$ represents the statistical significance relative to that of the GMtreated group at $p<0.05$.
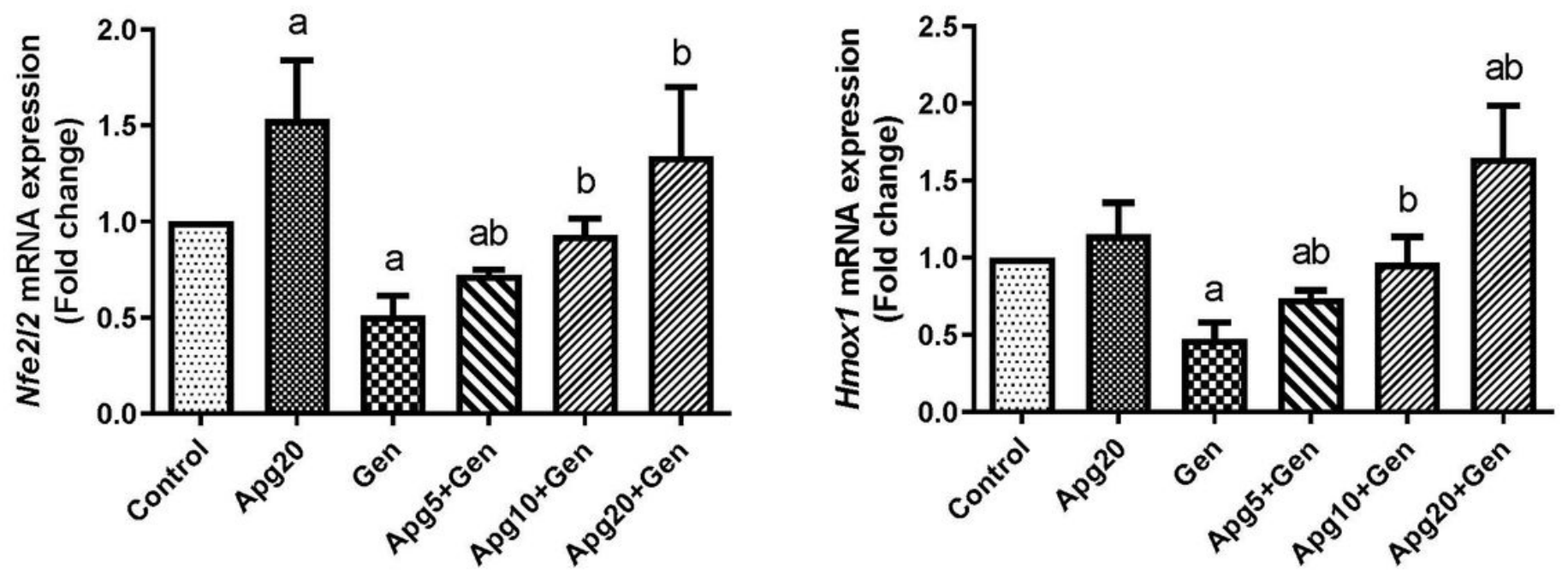

Figure 3

Renal mRNA expression of nuclear factor erythroid-2-related factor 2 and heme oxygenase 1 following apigenin treatment $\left(5,10\right.$, and $\left.20 \mathrm{mg} \mathrm{kg}^{-1}\right)$ in rats with gentamicin (GM)-induced nephrotoxicity. mRNA expression results are expressed as mean \pm standard deviation of three assays in duplicate references to $A c t b$ and represented as fold changes (log2 scale) compared with the mRNA levels of the control group. ${ }^{a}$ represents the statistical significance relative to that of the control group at $p<0.05 .{ }^{\mathrm{b}}$ represents the statistical significance relative to that of the GM-treated group at $p<0.05$. 

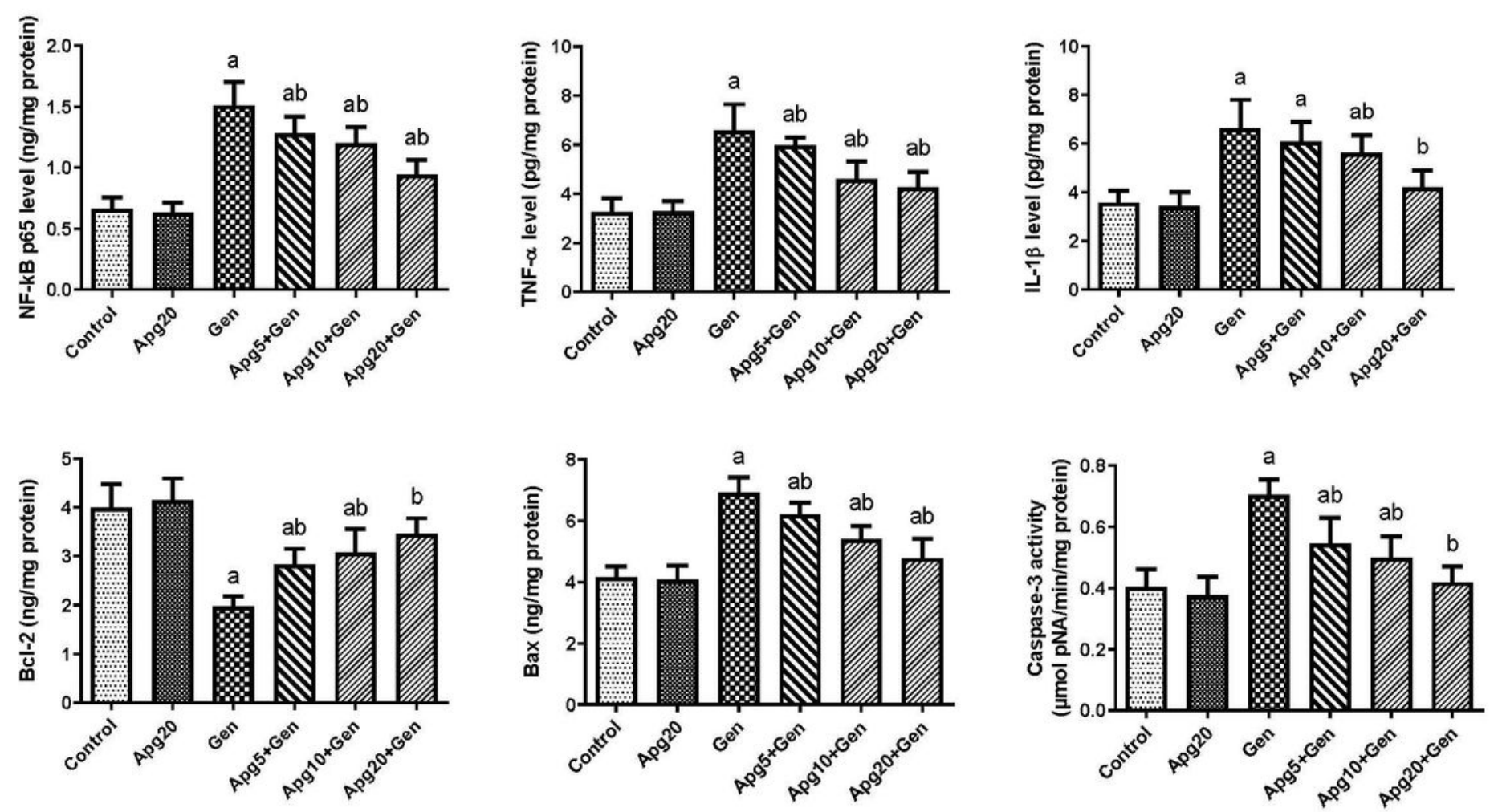

Figure 4

The renal levels of nuclear factor kappa B p65 subunit, tumor necrosis factor-alpha, and interleukin-1 $\beta$, and apoptotic-related protein levels (Bcl-2, Bax, and caspase-3) following apigenin treatment $(5,10$, and $20 \mathrm{mg} \mathrm{kg}^{-1}$ ) in rats with gentamicin (GM)-induced nephrotoxicity. Data are expressed as mean \pm standard deviation $(\mathrm{n}=7) .{ }^{a}$ represents the statistical significance relative to that of the control group at $p<0.05 .{ }^{b}$ represents the statistical significance relative to that of the GM-treated group at $p<0.05$.

\section{Figure 5}

Photomicrographs of kidney tissues from the control and apigenin-treated groups ( $A$ and $B$, respectively) show a normal kidney structure. In the gentamicin (GM)-treated rats (C), severe inflammation, cytoplasmic vacuolation, severe tubular necrosis and apoptosis, and congested glomeruli are shown. Pretreatment with apigenin $\left(5,10\right.$, and $\left.20 \mathrm{mg} \mathrm{kg}^{-1}\right)(\mathrm{D}, \mathrm{E}$, and $\mathrm{F}$, respectively) markedly attenuated all renal damages caused by GM. Hematoxylin and eosin, scale bar= $20 \mu \mathrm{m}$. 